\title{
SLAC T-510: Experimental validation of particle-level simula- tions of radio emission from particle cascades
}

\author{
K. Mulrey ${ }^{1, \star}$ for the $\mathrm{T}-510$ collaboration \\ ${ }^{1}$ Astrophysical Institute, Vrije Universiteit Brussel, Pleinlaan 2, 1050 Brussels, Belgium
}

\begin{abstract}
The SLAC T-510 experiment was designed to measure radio emission from particle cascades in a controlled laboratory setting and compare measurements with predictions from particle-level simulations. An electron beam incident upon a dense dielectric target produced a particle cascade in the presence of a strong magnetic field. We previously reported the agreement between data and simulations within systematic uncertainties, the largest being the reflection of radio emission within the target. A follow-up experiment has since been carried out to characterize the reflections and include them in simulations. In this contribution we report these new results. The uncertainties in the experiment are greatly reduced, and the features in the observed emission are well understood.
\end{abstract}

\section{Comparison between data and simulations}

Particle-level simulations are necessary for the interpretation of measured radio emission from extensive air showers. Several simulation packages are regularly used for this purpose, including CoREAS [1] and ZHAireS [2]. These simulations track radio emission from individual particles in the cascade, and so no assumptions are made about emission mechanisms. The SLAC T-510 experiment was designed to measure radio emission from particle cascades in a controlled laboratory setting and compare measurements with particle-level simulations. Simulations were done using the Geant4 [3] package to track particles in the cascade, and the endpoint [4] and ZHS [5] formalisms to calculate radio emission. The SLAC facility was used to provide electron beams that generated cascades in a high density polyethylene target. Radio emission propagated out of the target and to an array of dual-polarization antennas sensitive to the $200-1200 \mathrm{MHz}$ band. Coils placed underneath the target provided a variable, vertical magnetic field between \pm 970 Gauss. The experiment was designed so that emission due to charge excess effects would be isolated in the vertical polarization of the antenna, and emission due to magnetic effects would be isolated in the horizontal polarization. The antenna array was adjustable and sampled emission at a variety of vertical positions across the radiation pattern. Details about the experiment and simulations can be found in [6-8].

A comparison showed that simulations using both endpoint and ZHS formalisms were in agreement with each other, but produced pulse amplitudes that were $\sim 35 \%$ lower than measured data. The left panel of Figure 1 shows the horizontally polarized signal measured in the antenna at the point of peak emission, which was $652 \mathrm{~cm}$ above beam height. This discrepancy was attributed to reflections

^e-mail: kmulrey@vub.be 
off the bottom of the target. The reflection coefficient at this interface was previously unknown. In this contribution, we account for reflections in simulations, bringing data and simulation into agreement within systematic uncertainties.

There are a number of possible reflected paths in the target from which the emission can reach the antenna. The primary path follows emission that reflects off the bottom surface of the target. It is separated from the primary signal by $\sim 1 \mathrm{~ns}$. The second reflection first reflects off of the top of the target, and then the bottom. It is separated by $\sim 7 \mathrm{~ns}$. There are also higher order reflections that contribute to the measured signal. In order to include these reflections in simulations, the reflection coefficient of the bottom of the target must be known. This was measured in a dedicated experiment at Cal Poly in the fall of 2017. Results showed that the reflection coefficient was close to 1 over the frequency band.
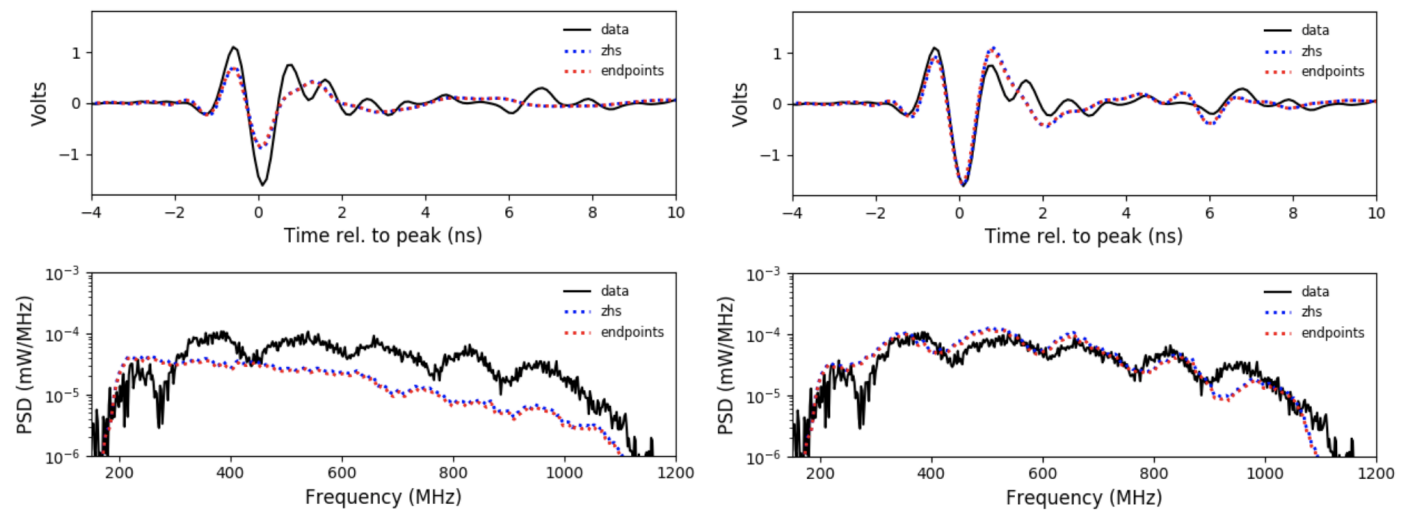

Figure 1. Horizontally polarized signal with $B=970 \mathrm{G}$ at an antenna position of $652 \mathrm{~cm}$ above the beam height. Left: Voltage (top) and power spectrum (bottom) for data and simulations without reflections included. Right: Voltage (top) and power spectrum (bottom) for data and simulations with the primary reflections included.

The reflections were added to the simulated signal in the time domain, using the same pulse shape as the primary signal, delayed in time, and with corrected transmission coefficients from the top of the target. The results of including the reflections to the horizontally polarized signal at the point of peak emission are shown in the right panel of Figure 1. The inclusion of the first reflection recovers the amplitude of the pulse, and the second reflection generates the interference pattern seen in data.

Figure 2 shows the mapping of emission for both polarizations by peak amplitude, where the height on the $\mathrm{x}$-axis refers to the height of the antenna above the beam. The inclusion of the reflections improves the agreement between data and simulation in both polarizations.

Another important measurement was the linearity of the strength of magnetically induced emission with the strength of the magnetic field. The ratio of horizontally to vertically polarized emission is shown in Figure 3. Again, including reflections in the simulations improves the agreement with data.

The SLAC T-510 experiment was designed to validate particle-level simulations. An initial comparison of simulations and data showed a discrepancy in signal amplitude, presumably due to reflections within the target. A follow-up experiment measured the reflection coefficient of the interface at the bottom of the target, confirming that it was close to one in the relevant frequency band. Including the reflections in the simulations brought data and simulation into agreement within systematic uncertainties. 

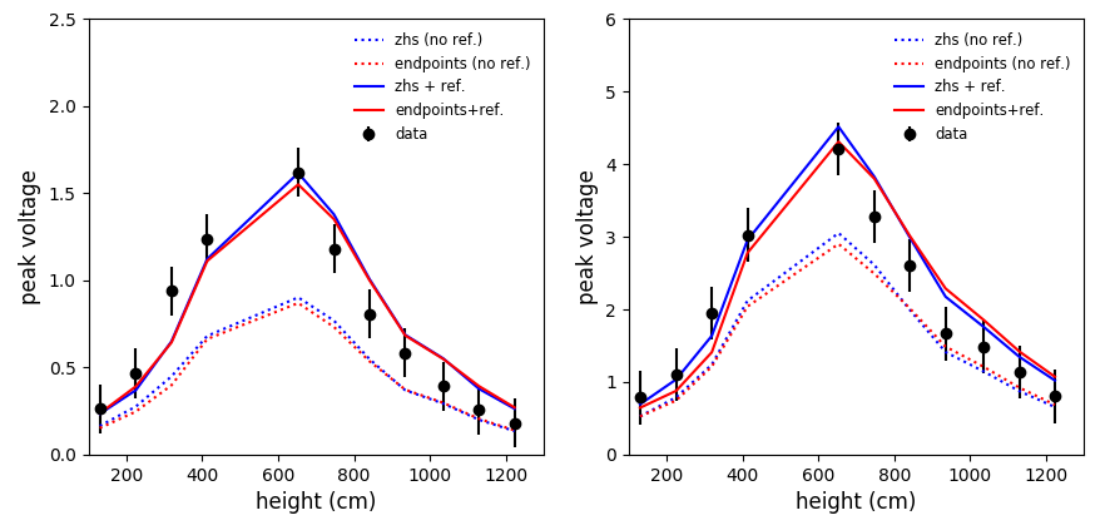

Figure 2. Radiation, mapped by peak amplitude of the signal, with a magnetic field strength of $B=970 \mathrm{G}$. Horizontally (magnetically induced) polarized emission is shown on the left, and vertically (charge excess induced) polarized emission is on the right. Data is shown in black, with systematic error bars. Simulations are shown with and without reflections added. The dashed line indicates simulations with reflections.

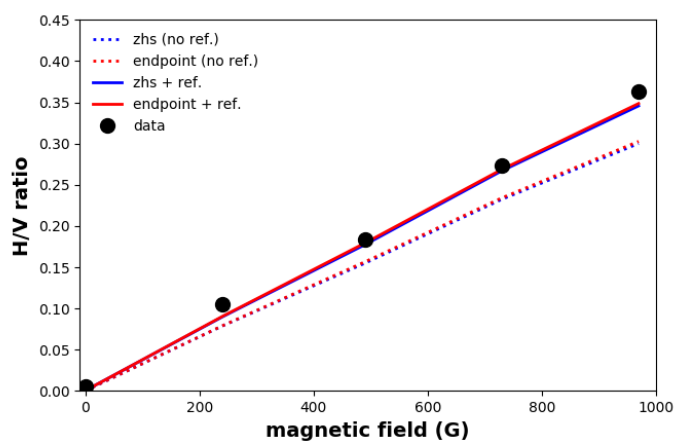

\section{References}

[1] T. Huege, M. Ludwig, C. James, AIP Conf. Proc. 1535, 128 (2013)

[2] J. Alvarez-Muñiz, W.C. Jr., E. Zas, Astropart. Phys. 35, 325 (2012)

[3] S. Agostinelli et al. (GEANT4), Nucl. Instrum. Meth. A506, 250 (2003)

[4] C. James, H. Falcke, T. Huege, M. Ludwig, Phys. Rev. E 84, 056602 (2011)

[5] E. Zas, F. Halzen, T. Stanev, Phys. Rev. D 45, 362 (1992)

[6] K. Belov et al. (T-510), Phys. Rev. Lett. 116, 141103 (2016)

[7] A. Zilles, EPJ Web Conf. 135, 01018 (2017)

[8] K. Mulrey, EPJ Web of Conf. 135, 01017 (2017)
Figure 3. The ratio of horizontally to vertically polarized signal is shown for an antenna $652 \mathrm{~cm}$ above beam height. Data is represented with black points. Simulations with and without reflections added are also shown, with the dashed line indicating the inclusion of reflections. 